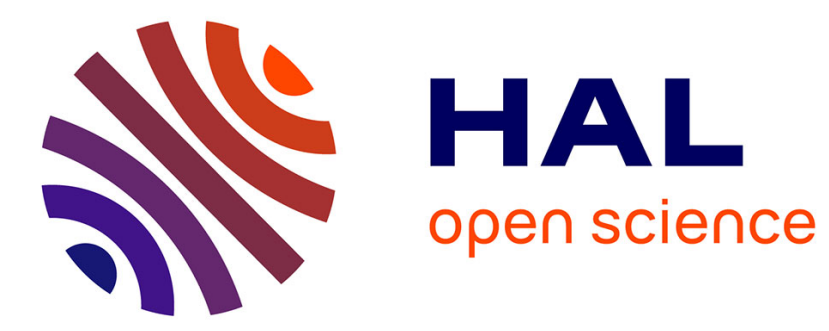

\title{
EFFECTS OF THE VERTICAL CoM MOTION ON ENERGY CONSUMPTION FOR WALKING HUMANOIDS
}

S Omran, S Sakka, Y Aoustin

\section{- To cite this version:}

S Omran, S Sakka, Y Aoustin. EFFECTS OF THE VERTICAL CoM MOTION ON ENERGY CONSUMPTION FOR WALKING HUMANOIDS. 17th International Confer ence on Climbing and Walking Robots (CLAWAR), Jul 2014, Poznan, Poland. 10.1142/9789814623353_0040 . hal-01199826

\section{HAL Id: hal-01199826 \\ https://hal.science/hal-01199826}

Submitted on 16 Sep 2015

HAL is a multi-disciplinary open access archive for the deposit and dissemination of scientific research documents, whether they are published or not. The documents may come from teaching and research institutions in France or abroad, or from public or private research centers.
L'archive ouverte pluridisciplinaire HAL, est destinée au dépôt et à la diffusion de documents scientifiques de niveau recherche, publiés ou non, émanant des établissements d'enseignement et de recherche français ou étrangers, des laboratoires publics ou privés. 


\title{
Effects of the vertical CoM motion on energy consumption for walking humanoids
}

\author{
S. OMRAN and S. SAKKA and Y. AOUSTIN* \\ Research Institute for Communications and Cybernetics of Nantes (IRCCyN), \\ 1 rue de La Nö̈, 44321 Nantes/France \\ *E-mail: sahab.omran,sophie.sakka,yannick.aoustin@irccyn.ec-nantes.fr
}

\begin{abstract}
This paper proposes an analysis of the effect of vertical motion of the CoM during humanoid walking. The linear inverted pendulum (LIP) model is classically used to deal with humanoid balance during walking. The effects on energy consumption of the CoM height remaining constant for humanoid robots, or varying like human beings, are badly known and are studied here. Two approaches are introduced for the comparison: the LIP which offers the great advantage of analytical solving (i.e. fast and easy calculations), and a numerical solving of the IP dynamics, which allows varying the height of the center of mass during walking. The results are compared in a dynamics simulation of the humanoid robot ROMEO (Aldebaran Robotics).
\end{abstract}

Keywords: humanoid; dynamics; inverted pendulum; numerical resolution; energy consumption; CoM vertical displacement, walking.

\section{Introduction}

The locomotion of humanoid robots is one of the most important topics in humanoid robotics. These highly advanced robots are expected to coexist with humans. For this reason, we aim to obtain a human-like walking. Vukobratovic et al. ${ }^{1}$ originally suggested the zero moment point (ZMP) as a criterion to study the dynamic stability of a biped robot. This criterion was widely used in the control of biped robots stability. Many researches proposed to first to generate the robot motion, and then to guarantee the stability by correcting the horizontal motion of the upper body. ${ }^{2,3}$ Nagasaka et $a l .{ }^{4}$ proposed an optimal method to minimize the error between the actual ZMP and its desired trajectory. Dasgupta et al..$^{5}$ suggested to use the joint variables captured from human subjects walking, but the horizontal motion of the trunk is modified before delivering it to the robot in order to maintain the ZMP close to its predefined reference trajectory. 
Other ZMP-depending methods represent the robot by an inverted pendulum. These methods specify the ZMP trajectory in advance and then calculate the center of mass (CoM) motion corresponding to the desired ZMP. ${ }^{6,7}$ Yuan et al. ${ }^{8}$ also uses the inverted pendulum equation to define the horizontal displacement of the waist. He considers a very small-amplitude vertical motion of the center of mass but he does not take it into account when calculating the horizontal motion of CoM in order to simplify the system.

However, most of the previous methods do not take into account the vertical motion of the center of mass and this leads in most cases to a not very human-like walking gait. In addition, robots walk with their knees bent which makes the system consume more energy. ${ }^{9,10}$ In this paper we suggest to solve numerically the differential equation relying the horizontal components of the center of mass of the pendulum and the ZMP components. In fact, the numerical solution allows us to consider the vertical displacement of the robot CoM because the mathematical complexity of this equation is no longer troubling. And this allows us to generate a more human-like walking gait because the waist oscillates with a smaller magnitude and moves also vertically. Finally, our system with a 3D-displacement of CoM consumes fewer energy than the one with a constrained vertically CoM.

\section{Inverted pendulum dynamics equations}

The dynamics of a humanoid robot can be approximated by the inverted pendulum model. This approximation leads to a relation linking the CoM position to the ZMP position, which can be expressed in 3D as

$$
x_{p}=x-\frac{z-z_{P}}{\ddot{z}+g} \ddot{x} \quad ; \quad y_{p}=y-\frac{z-z_{P}}{\ddot{z}+g} \ddot{y}
$$

where $P=\left[\begin{array}{lll}x_{P} & y_{P} & z_{P}\end{array}\right]^{t}$ and $C=\left[\begin{array}{lll}x & y & z\end{array}\right]^{t}$ denote the ZMP and CoM positions in the reference frame, respectively. The differential equations system (1) is non linear and difficult to solve analytically.

\subsection{Analytical solving}

A solution to use these equations for humanoid control consists in linearizing the system in $x$ and $y$. Kajita et al. considered that the vertical motion of the CoM is fixed with $z=z_{c}$, therefore $\ddot{z}=0$ the. ${ }^{11,12}$ The differential system (1) becomes linear in $x$ and $y$ :

$$
x_{p}=x-\frac{z_{c}-z_{P}}{g} \ddot{x} \quad ; \quad y_{p}=y-\frac{z_{c}-z_{P}}{g} \ddot{y}
$$


The differential system (2) accepts a solution of the form:

$$
\left\{\begin{array}{l}
x=x(0) \cosh \left(t / T_{c}\right)+T_{c}\left(\dot{x}_{0}\right) \sinh \left(t / T_{c}\right) \\
y=y(0) \cosh \left(t / T_{c}\right)+T_{c}\left(\dot{y}_{0}\right) \sinh \left(t / T_{c}\right)
\end{array}\right.
$$

Where $T_{c}=\sqrt{\frac{z_{c}}{g}}$ and $z_{c}$ is the constant height of the robot center of mass. This solution is rather simple, but does not lead to a very human-like gait as the waist of the robots remains at the same height during its gait.

\subsection{Numerical solving}

The vertical variation of the CoM position will be an input. We will, represent the vertical component of the CoM position as a function of time: $z=z(t)$. The function $z(t)$ is set depending on the description of the vertical displacement of the CoM for human walking defined by Hayot et al. ${ }^{13}$ According to this study $z(t)$ should be a function oscillating along the average height of the CoM $z_{c}$, reaching its maximal value at midstance and its minimal value at the middle of the double support phase. A function of the form (4) can meet the description of $z(t)$, it is illustrated in Fig. 1:

$$
z=z_{c}+A \cos (\omega t+\phi)
$$

Where $A$ denotes the vertical amplitude of the CoM altitude; $\omega=2 \pi / T$; $T$ is the period of one step; $\phi$ is some angle depending on the first phase of the motion (Single Support SS or Double Support DS).

The resulting $\ddot{x}$ is obtained by reporting $z$ and $\ddot{z}$ in Eq.(1).

$$
\ddot{x}=\frac{g-\omega^{2} A \cos (\omega t+\phi)}{z_{c}+A \cos (\omega t+\phi)}\left(x-x_{p}\right)
$$

The expression of $\ddot{y}$ may be obtained using the same treatment. But we will limit our study to the 2D motion in the sagittal plane. However, the differential system leading to 5 is non-linear and non-homogeneous of second order. If not linearized, no analytic solution exist for these equations.

\section{Modeling}

\subsection{Walking cycle of the humanoid robot}

The walking cycle is defined by two successive steps (right and left legs). One step is composed of a single support phase (SS) on the stance leg, delimited by swinging foot takeoff and swinging foot strike, and a double support phase (DS) where balance is distributed on both legs, delimited by swinging foot strike and the other leg foot takeoff. The system parameters 


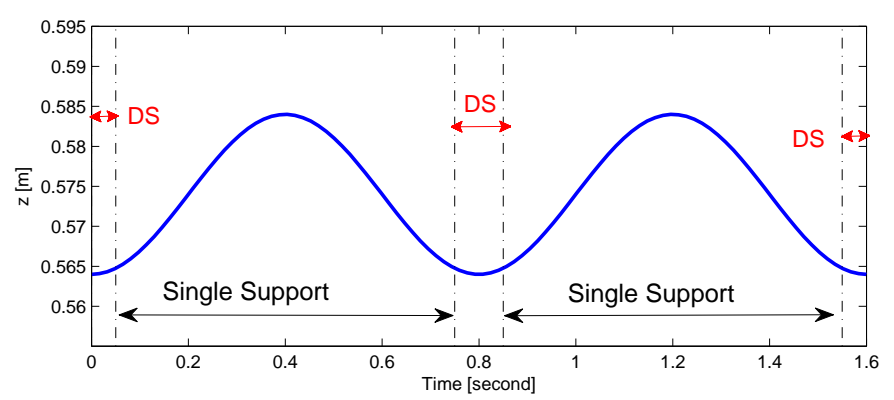

Fig. 1. desired vertical motion of the robot center of mass during one walking cycle, using a sinusoidal function.

for positions, velocities and accelerations are equal at the beginning and at the end of each cycle. In what follows, the following hypotheses will be considered for the complete motion: The feet soles remain parallel to the ground; The trunk segment remains vertical; CoM and waist segment have the same velocity profiles; Feet velocity and acceleration are equal to zero at foot strike.

\subsection{Studied robot}

Let us consider the $2 \mathrm{D}$ humanoid robot shown in Fig. 2 . It is composed of 6 actuators ( 2 ankles, 2 knees and 2 hips) to control its body movements in the sagittal plane. Let $\mathbf{q}$ denote the system generalized coordinates vector.

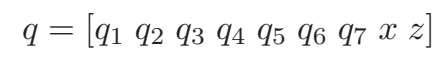

Where $q_{1} q_{2} \ldots q_{7}$ are the joint variables and $x z$ are the Cartesian coordinates of the hip. The reference frame is defined such that $\mathbf{x}_{0}$ denotes the horizontal advancement direction, $\mathbf{z}_{0}$ is the vertical bottom-up direction, and $\mathbf{y}_{0}=\mathbf{z}_{0} \times \mathbf{x}_{0}$ is the lateral direction.

\subsection{Dynamic model}

The dynamics of the system may be described by the following equations.

$$
\begin{cases}D \ddot{\mathbf{q}}+C \dot{\mathbf{q}}+G=B \boldsymbol{\Gamma}+J_{i}^{t} \mathbf{R}_{i} & \text { if in single support, } \operatorname{leg}_{i} \\ D \ddot{\mathbf{q}}+C \dot{\mathbf{q}}+G=B \boldsymbol{\Gamma}+J_{1}^{t} \mathbf{R}_{1}+J_{2}^{t} \mathbf{R}_{2} & \text { if in double support }\end{cases}
$$

The matrices $\mathbf{D}, \mathbf{C}$, and $\mathbf{G}$ describe respectively the inertia, Coriolis and gravity forces acting on the system. The matrix $\mathbf{B}$ is the actuation matrix; 


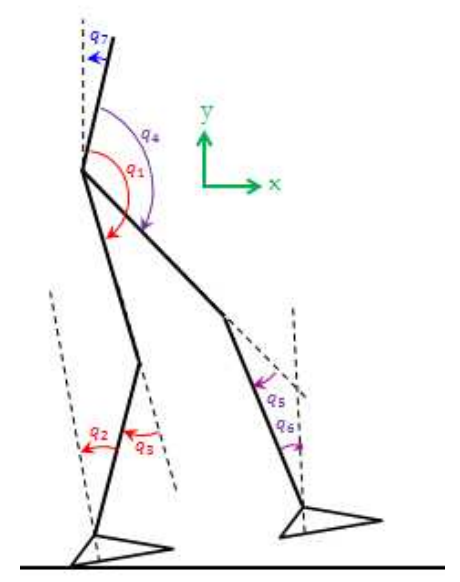

Fig. 2. The 2D studied robot.

it expresses the contribution of each joint torque in the virtual work $\delta w$ :

$$
\delta w=\Gamma_{1} \delta q_{1}+\Gamma_{2} \delta q_{2}+\ldots+\Gamma_{6} \delta q_{6}=\delta \mathbf{q}^{t} B \boldsymbol{\Gamma}
$$

where $\boldsymbol{\Gamma}=\left[\begin{array}{llll}\Gamma_{1} & \Gamma_{2} & \ldots & \Gamma_{6}\end{array}\right]^{t}$ and $B=\left[\begin{array}{ll}I_{6} & 0_{6 \times 3}\end{array}\right]^{t}$. The vectors $\mathbf{R}_{1}$ and $\mathbf{R}_{2}$

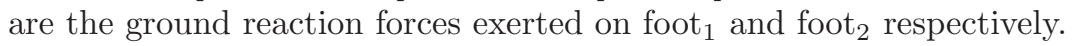

$$
\mathbf{R}_{1}=\left[\begin{array}{lll}
R_{1 x} & R_{1 z} & M_{1 y}
\end{array}\right]^{t} \quad \mathbf{R}_{2}=\left[\begin{array}{lll}
R_{2 x} & R_{2 z} & M_{2 y}
\end{array}\right]^{t}
$$

In single support, there are $n=9$ unknown variables which are the components of $\left(\boldsymbol{\Gamma}, \mathbf{R}_{1}\right)$ or $\left(\boldsymbol{\Gamma}, \mathbf{R}_{2}\right)$ depending on which foot is in contact with the ground. So, the $n$ independent equations in the first line of (6) are sufficient. On the other hand, in double support there are 12 unknown variables in $\left(\boldsymbol{\Gamma}, \mathbf{R}_{1}, \mathbf{R}_{2}\right)$ and only $n=9$ equations available. In order to solve the problem in double support, 3 variables should be chosen and set to completely describe the system dynamics. Let us fix the 3 components of ground reaction forces exerted on the foot that was swinging before the considered double support.

Let $\operatorname{leg}_{1}$ be the stance leg before the considered double support, and $\operatorname{leg}_{2}$ the swinging leg. In the single support just after the considered double support, the stance and swinging legs are exchanged. The first step consists in solving the dynamics for both single support phases. So we obtain the joint torques and the ground reaction forces in the both single support phases. Then the 3 components of $R_{2}$ can be defined by a third order polynomial meeting the boundary conditions : 
- $R_{2}$ at the beginning of the $D S=R_{2}$ at the end of the previous $S S$.

- The derivative of $R_{2}$ at the beginning of the $D S=$ the derivative of $R_{2}$ at the end of the previous $S S$.

- $R_{2}$ at the end of the $D S=R_{2}$ at the beginning of the next $S S$.

- The derivative of $R_{2}$ at the end of the $D S=$ the derivative of $R_{2}$ at the beginning of the next $S S$.

Then the joint torques and $R_{1}$ can be deduced from the second line of Eq. (6) :

$$
\left[\begin{array}{c}
\boldsymbol{\Gamma} \\
\mathbf{R}_{1}
\end{array}\right]=\left[B J_{1}^{t}\right]\left[D \ddot{\mathbf{q}}+C \dot{\mathbf{q}}+G-J_{2}^{t} \mathbf{R}_{2}\right]
$$

Figure 3 illustrates two components of $\mathbf{R}_{1}$ and $\mathbf{R}_{2}$ for two walking cycles. The blue part of the curves result from dynamics equations, while the red parts match the rebuilt components during the double supports. A similar process is performed for all double support phases.
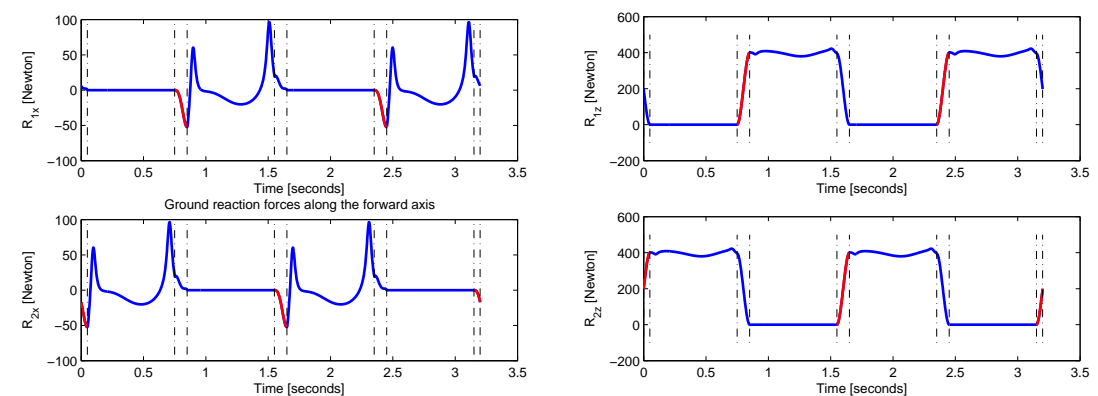

Fig. 3. Evolution of ground reaction forces $R_{x}$ (left) and $R_{z}$ (right).

\section{Results}

\section{Simulation settings}

We have used the model of the humanoid robot described in 3.2 to realize a walking gait according to the the hypotheses mentioned in 3.1.The desired trajectory of the ZMP was defined as a polynomial function of time (Fig. 4). The step length was set to $0.348[\mathrm{~m}]$ and the cycle duration to $1.6[\mathrm{~s}]$, with $0.1[\mathrm{~s}]$ for each double support and $0.7[\mathrm{~s}]$ for each single support phases. The resulting CoM forward displacement is shown in Fig. 4. It was then 
calculated using either the linear inverted pendulum (2) or the numerical solving of (1). We note that the two forward CoM trajectories are almost identical. Then the joint variables were calculated using the inverse kinematics. In the following figures, the single and double support phases are delimited by vertical dashed lines.

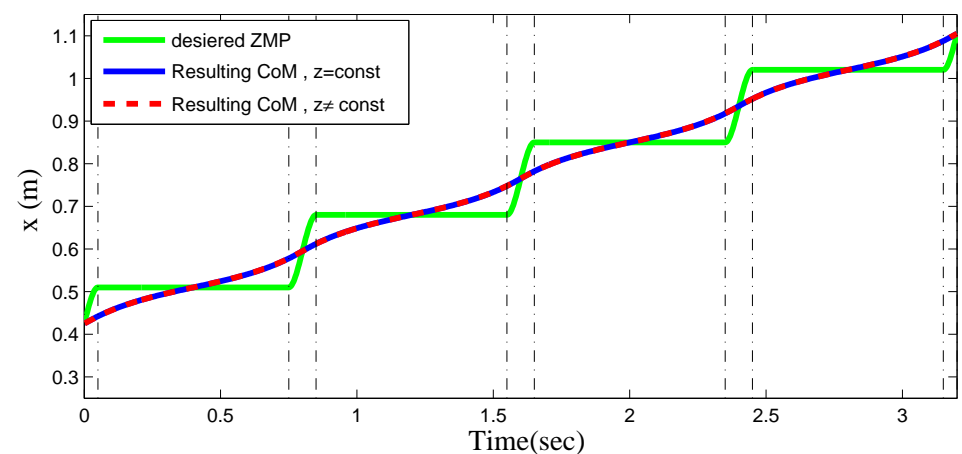

Fig. 4. Desired ZMP trajectory for two walking cycles and the resulting CoM trajectory.

\section{Joint torques}

In the following, the results of the dynamic model are illustrated for two cases: when the robot walks with a fixed height of its center of mass $(A=0)$ and when the robot walks with a sinusoidal height of its center of mass with amplitude of $(A=2[\mathrm{~cm}])$. From figure 5 we notice that the joint torques at knees and ankles are smoother when the CoM of the robot oscillates sinusoidally in the vertical direction.

\section{Energy consumption}

Many criteria exist to evaluate energy consumption of a mechanical system. In this approach, we chose the sthenic criterion $E$ which is defined as the quadratic actuated torque per unit of distance, as exposed in eq (8).

$$
E=\frac{1}{d} \int_{t_{0}}^{t_{f}} \Gamma^{t} \Gamma d t
$$

where $t_{0}$ and $t_{f}$ denote the beginning and ending instants of the total observed motion, $d$ is the traveled distance. Fig. 6 (a) illustrates the sthenic criterion $E$ as a function of the amplitude $2 A$ of the CoM sinusoidal trajectory for three values of $z_{c}$, the mean height of CoM. We notice that the 

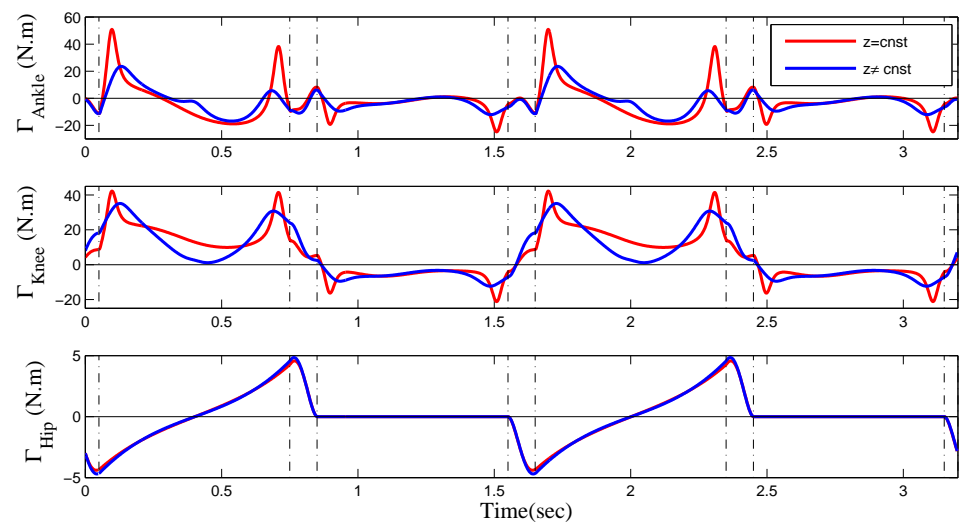

Fig. 5. Comparison of pitch ankle, knee and hip torques evolution when the CoM height is constant (red curves) or varying with $A=2[\mathrm{~cm}]$ (blue curves) for one leg during two cycles of walking.

sthenic criterion decreases considerably when $A$ increases in the range $[0,2$ $\mathrm{cm}]$. The quadratic actuated torque value $E_{0}=\Gamma^{t} \Gamma$ with time is illustrated in Fig. 6 (b). We notice that $E_{0}$ is very high at both ends of single support phases, for the two cases : $z=$ cnst and $A=2[\mathrm{~cm}]$. But $E_{0}$ is less whit $A=2 C[\mathrm{~cm}]$ than with $z=$ cnst. This effect comes from the fact that the knee is less bended with a CoM height variation. As a consequence, the lever is also reduced, and the required torque decreases. When $A$ is greater than certain value depending on the $z_{c}$ (about $2[\mathrm{~cm}]$ for the studied structure), $E$ starts to increase. So that, depending on $z_{c}$, there is a limit of $A$ that we can not exceed because the knee angle comes close to $\pi$ in midstance.

\section{5. conclusion}

This paper proposed an analysis of the effect of CoM vertical magnitude on the energy consumption for humanoid walking gait. A 2D simulation was proposed to compare the classical LIP model solved analytically to an IP model solved numerically and offering a possible variation of the CoM height during walking. The dynamics analysis allowed to come out with the following conclusions:

(1) For both IP models, the highest actuator torques occur at the change of single and double support phases;

(2) The use of a variable CoM height considerably reduces the torque so- 


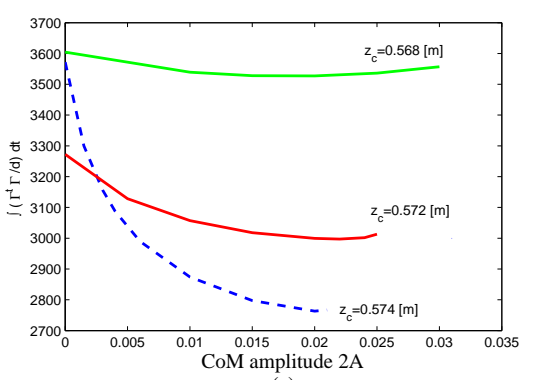

(a)

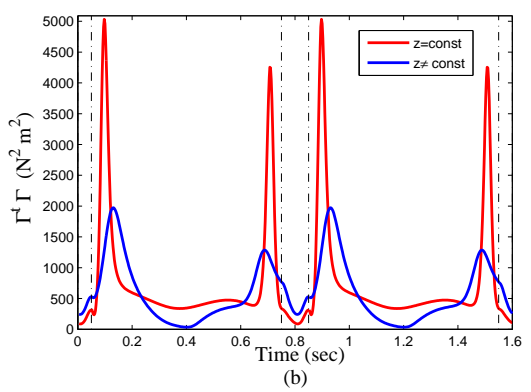

Fig. 6. Sthenic criterion

licitations at the change of support, and at midstance;

According to this conclusions, graphs giving the optimal value of the CoM vertical magnitude according to step length and walking velocity may be obtained and included in humanoid walking gait generators to minimize the energy consumption.

\section{References}

1. M. VUKOBRATOVIC, A. Frank and D. Juricic, On the stahility of biped locomotion, in IEEE TRANSACTIONS ON BIO-MEDICAL ENGINEERING, 1970.

2. K. Nishiwaki, Y. Kagami, S.and Kuniyoshi, M. Inaba and H. Inoue, Online generation of humanoid walking motion based on a fast generation method of motion pattern that follows desired zmp, in IEEE/RSJ International Conference on Intelligent Robots and Systems (IROS), 2002.

3. Q. Li, A. Takanishi and I. Kato, Learning control for a biped walking robot with a trunk, in Proceedings of 1993 IEEERSJ International Conference on Intelligent Robots and Systems IROS 93, 1993.

4. K. Nagasaka, H. Inoue and M. Inaba, Dynamic walking pattern generation for a humanoid robot based on optimal gradient method, in IEEE, 1999.

5. A. Dasgupta and Y. Nakamura, Making feasible walking motion of humanoid robots from human motion capture data, in IEEE International Conference on Robotics and Automation, 1999.

6. K. Harada, s. Kajita, K. Kaneko, and H. H, World scientifique-International Journal of Humanoide Robotiues 16, 640 (October 2004).

7. S. Kajita, F. Kanehiro, K. Kaneko, K. Yokoi and H. Hirukawa, Hirukawa, The $3 \mathrm{~d}$ linear inverted pendulum mode: A simple modeling biped walking pattern generation, in Proceedings of International Conference on Intelligent Robots and Systems, 2001.

8. Q. Yuan and I.-M. Chen, Human-like walking of humanoid robot based on 
biped kinematics and captured motion of human, in 13th World Congress in Mechanism and Machine Science, June 2011.

9. y. Ogura, T. Kataoka, K. Shimomura, H. Lim and A. Takanishi, A novel method of biped walking pattern generation with predetermined knee joint motion, in Procwdings of 2004 IEEEIRSJ International Conference on Intelligent Robots and Systems, September 28 -October 2004.

10. Y. Ogura, K. Shimomura, H. Kondo, A. Morishima, T. d. Okubo and S. Momoki, Human-like walking with knee stretched, heel-contact and toe-off motion by a humanoid robot, in Proceedings of the 2006 IEEE/RSJ International Conference on Intelligent Robots and Systems, October 2006.

11. S. Kajita, K. Yokoi, M. Saigo and K. Tanie, Balancing a humanoid robot using backdrive concerned torque control and direct angular momentum feedback, in IEEE International Conference on Robotics and Automation, 2001.

12. S. Kajita, F. Kanehiro, K. Kaneko, K. Fujiwara, K. Harada, K. Yokoi and H. Hirukawa, Resolved momentum control: Humanoid motion planning based on the linear and angular momentum, in IEEE/RSJ International Conference on Intelligent Robots and Systems, 2003.

13. C. Hayot, S. Sakka and P. Lacouture, Gait \& Posture submitted, p. . (2010). 